\title{
Nematic effects and strain coupling in entangled polymer melts under strong flow
}

Kirkensgaard, Jacob J K; Hengeller, Ludovica; Dorokhin, Andriy; Huang, Qian; Garvey, Christopher J.; Almdal, Kristoffer; Hassager, Ole; Mortensen, Kell

Published in:

Physical Review E

Link to article, DOI:

10.1103/PhysRevE.94.020502

Publication date:

2016

Document Version

Peer reviewed version

Link back to DTU Orbit

Citation $(A P A)$ :

Kirkensgaard, J. J. K., Hengeller, L., Dorokhin, A., Huang, Q., Garvey, C. J., Almdal, K., Hassager, O., \& Mortensen, K. (2016). Nematic effects and strain coupling in entangled polymer melts under strong flow. Physical Review E, 94(2), [020502]. https://doi.org/10.1103/PhysRevE.94.020502

\section{General rights}

Copyright and moral rights for the publications made accessible in the public portal are retained by the authors and/or other copyright owners and it is a condition of accessing publications that users recognise and abide by the legal requirements associated with these rights.

- Users may download and print one copy of any publication from the public portal for the purpose of private study or research.

- You may not further distribute the material or use it for any profit-making activity or commercial gain

- You may freely distribute the URL identifying the publication in the public portal 


\title{
Nematic effects and strain coupling in entangled polymer melts under strong flow
}

\author{
Jacob J.K. Kirkensgaard ${ }^{a *}$, Ludovica Hengeller ${ }^{c}$, Andriy Dorokhin ${ }^{b}$, Qian Huang $^{c}$, \\ Christopher J. Garvey ${ }^{d}$, Kristoffer Almdal ${ }^{b}$, Ole Hassager ${ }^{c}$ and Kell Mortensen ${ }^{a *}$ \\ ${ }^{a}$ Niels Bohr Institute, University of Copenhagen, Denmark \\ ${ }^{c}$ Department of Chemical and Biochemical Engineering, Technical University of Denmark \\ ${ }^{b}$ Department of Micro- and Nanotechnology, Technical University of Denmark and \\ ${ }^{d}$ Bragg Institute, Australian Nuclear Science and Technology Organisation, Lucas Heights, NSW 2234, Australia
}

(Dated: August 4, 2016)

\begin{abstract}
We use small-angle neutron scattering (SANS) to study labeled short chains with and without the influence of an entangled and highly stretched surrounding environment of longer chains. We find unequivocal evidence of nematic effects as the blend chains in steady state flow are stretched a factor $\sim 1.5$ more from the presence of the long chain nematic field. In the pure melt we confirm that the non-affine mean-field result $\nu=0.5$ for the strain coupling is still valid for very fast flows, while in the nematic system our analysis predict an increased coupling constant. We provide a structural explanation for the two first regimes of the non-linear relaxation, particularly a transition regime where the long chains are relaxing in a sea of reptating short chains.
\end{abstract}

Filament stretching rheometry (FSR) allows large deformation up to Hencky strain 7 (stretch ratio 1000) with accurate constant stretch rates and subsequently keeping the large deformation state during relaxation of the sample. These unique features of the FSR therefore open up for new investigations in large strain polymer dynamics $[1,2]$. Despite the recognized success of the classical DoiEdwards tube model [3] and its later modified manifestations [4], the nonlinear viscoelastic behaviour of entangled polymer chains in fast flows still possess a challenge and there is currently no full theoretical description of the underlying physics in such systems. One novel parameter in the tube model, proposed very recently in both simulations $[5,6]$ and experiments $[7,8]$, is the anisotropic friction due to nematic interactions between polymer-polymer (long chain-long chain) and polymeroligomer (long chain-short chain). However, the possible changes of molecular configurations resulting from such nematic interactions, which may be seen in SANS, have not yet been systematically investigated. Here we push the idea of an entangled polymer system diluted by shorter chains into the regime of a bidisperse melt of long and short entangled chains and flip the viewpoint by focusing on the short chain behaviour and how these are influenced by the presence of a highly entangled and stretched surrounding environment of longer chains. We exploit the power of FSR to perform true stress relaxation experiments after steady state flow and combine these with SANS experiments to obtain unique structural information on the short chain conformations during relaxation with and without the influence of the stretched long chain environment. We investigate two systems: a pure melt of short polystyrene $(95 \mathrm{~kg} / \mathrm{mol})$ chains and a bidisperse melt composed of a 50/50 wt mixture of short and long $(545 \mathrm{~kg} / \mathrm{mol})$ polystyrene chains. Note that both chain populations are entangled with the number of entanglements per chain $Z_{L} \approx 41$ and $Z_{S} \approx 7$ for the long and short chains respectively [10]. In both systems a fraction of the short chains are deuterated and thus allows for direct comparison of the short chain relaxation in these two scenarios. We label these two sample series Short-in-Short (SiS) and Short-in-Long (SiL) respectively. Synthesis and chromatography of the monodisperse polystyrenes, PS-545k and PS-95k used in this work have already been described along with characterisations of both shear and extensional rheology $[10,11]$. The main characteristics of the sample constituents are summarised in Table I. As shown in [10] extensional steady state flow conditions are established beyond a Hencky strain of $\epsilon=3$ and a total of five strain rates were rheologically tested probing different flow regions separated by the time constants of the constituent chains. Here we focus our attention to $\epsilon=3$ and to the highest strain rate investigated, $\dot{\epsilon}=0.1 \mathrm{~s}^{-1}$. We note that we also find nematic effects at lower strain rates which will be presented elsewhere. The details of the stretching experiments are given in [10]. However, for the SANS experiments it is vital to quench the samples fast enough to trap the relevant molecular configurations. Stretching is performed at $130^{\circ} \mathrm{C}$ and our procedure results in a cooling rate of ca. $10 \mathrm{~K} / \mathrm{s}$ ensuring that within $\sim 3 \mathrm{~s}$ the sample is below its glass transition temperature [12]. This time is much smaller than the Rouse time of the short polymer chains $(\sim 20 \mathrm{~s})$, so we are confident that the initial molecular configuration survives. This is confirmed by scattering experiments on samples quenched at a lower temperature $\left(125^{\circ} \mathrm{C}\right.$, but at the same Weissenberg number) which within experimental error are identical to the $130^{\circ} \mathrm{C}$ results. Figure 1 shows the stress relaxation data for the SiL and SIS samples normalized by the first value of the stress decay $\sigma(t=0)$ plotted against time where $t=0$ is the steady state and the start of the relaxation part of the experiment. The red arrows highlight the times at which a quenching was performed and thus six different stages of the relaxation process have been investigated. In the figure the Rouse and reptation time 
of the monodisperse linear short components are indicated. In [10] the rheology data of the blend is separated into 3 domains approximately defined in the following time intervals: a fast regime $(0-20 \mathrm{~s})$, a transition regime (20-700s) and a slow regime for times longer than 700s. It is hypothesised that the fast regime is dominated by fast stretch relaxation (note that the blend initially relaxes faster than the pure short chain melt) and the slow regime by long chain relaxation in a sea of essentially relaxed short chains. The transition regime is speculated to originate from retraction of long chains in a sea of reptating short chains. Here we aim to clarify the structural origin of this rheological behaviour using SANS.

TABLE I: The weight-average molecular weight $\bar{M}_{w}$, the polydispersity index $P D I$ and the weight fractions of the polymers used in the samples SiL (Short in Long) and SiS (Short in Short).

\begin{tabular}{l|cccc}
\hline Components & PS-545k & PS-95k & D-PS-86k & D-PS-80k \\
\hline $\bar{M}_{w}[\mathrm{~g} / \mathrm{mol}]$ & 545000 & 95100 & 86300 & 80000 \\
$P D I$ & 1.12 & 1.07 & 1.02 & 1.02 \\
Sample SiL $(w t \%)$ & 50 & 40 & 10 & 0 \\
Sample SiS $(w t \%)$ & 0 & 90 & 0 & 10 \\
\hline
\end{tabular}

SANS originate from different beamlines. First experiments were performed at the SANS-1 instrument at the Swiss Spallation Neutron Source (SINQ) and the second at the Quokka beamline at ANSTO, Australia. In both cases 3 overlapping settings covered a full $q$-range from $5 \cdot 10^{-3} \AA^{-1}$ to $0.25 \AA^{-1}$. We followed standard data reduction procedures, i.e. correcting for detector efficiency using incoherent water scattering, and subtracting background scattering which is dominated by the incoherent scattering from polystyrene and measured in a polystyrene sample with no deuterated chains. Here we focus on the central setting for $2 \mathrm{D}$ fitting since the relevant length scales are best represented here. Our anisotropic SANS data can be modelled using the Warner-Edwards (WE) model [13] for the SiS sample and the modified WE-model with dangling ends (DE-model) [14] which we find to be directly applicable for the SiL system without the complications arising from chain scission etc. present in a cross-linked system [1517]. As shown below the SiL data show distinct lozenge patterns while the SiS samples do not. The appearance of lozenges requires some chains to remain stretched while others relax, i.e. it requires an effective network, either in the form of a crosslinked system as was the subject in the original DE-papers $[14,15]$ or of a sufficiently longlived entanglement network which is thus the case in the SiL sample, but, according to our data, not in the SiS sample. In the DE-model each chain is described as having two dangling ends each a fraction $f$ of the total chain. The total scattering function is a combination of terms from the isotropic dangling ends described by

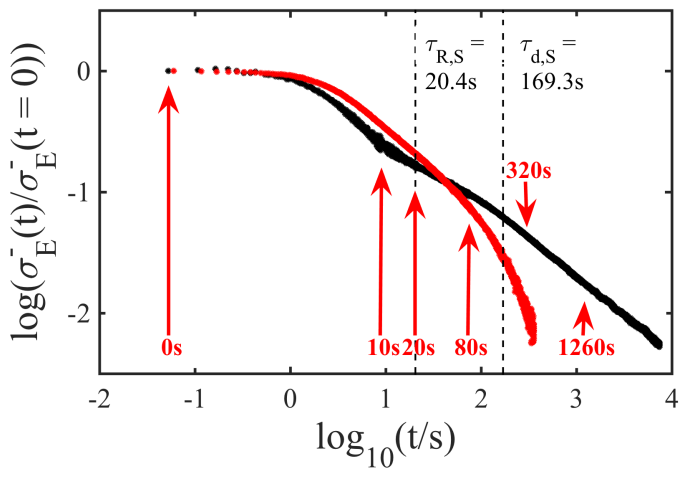

FIG. 1: Tensile stress $\sigma_{E}^{-}$of the SiL (black) and SiS (red) samples at $130^{\circ} \mathrm{C}$ after cessation of fast uniaxial elongational flow at Hencky strain $\epsilon=3$ and strain rate $\dot{\epsilon}=0.1 \mathrm{~s}^{-1}$. Dashed vertical lines indicate the short chain Rouse and reptation time. Red arrows indicate times where a quenced samples was produced for scattering studies.

Gaussian chain statistics and the central stretched portion described by the WE model. Thus, the DE model reduces to the WE model for $f=0$. In both models the tube diameter is given by $d_{\mu}=d_{0} \lambda_{\mu}^{\nu}$ with $\lambda_{\mu}$ being the microscopic strain ratio in direction $\mu(\mu=x, y, z)$ where $z$ is the stretch direction, $d_{0}$ the tube diameter of the relaxed melt and the $\nu$ parameter allowing for anisotropic strain coupling of the tube potential. Assuming incompressibility, the perpendicular strain ratios are related to $\lambda_{z}$ as $\lambda_{x}=\lambda_{y}=1 / \sqrt{\lambda_{z}}$. It is important to recognise that the microscopic and macroscopic strain ratios are not necessary the same [18] and a number of models have attempted to relate the two theoretically [19]. In particular, the value of the strain coupling has been a topic of discussion for some time [14, 20, 21] but for moderate strain ratios has been clearly demonstrated experimentally to be non-affine with $\nu=0.5$ [21] confirming various theoretical predictions $[22,23]$. However, it is unclear if this scaling also applies for the much higher strain ratio described here and how the exponent would change in a system influenced by nematic effects. Further, it is not clear how $\nu$ and (the effective microscopic) $\lambda_{z}$ behaves as the system relaxes. In our experiments, the macroscopic strain ratio experienced in the filament plane of observation at $t=0$ for the employed Hencky strain is $\lambda_{z, \text { mac }}=\exp (3) \approx 20$. Notice that this is considerably higher than any previously reported elongation ratio in SANS based structural studies of fully labeled chains to our knowledge a factor of 4-5 higher. We present fits where we allow both $\nu$ and $\lambda_{z}$ to vary, but impose constraints by the following strategy: Each consecutive fit uses the fit of the previous time point as lower/upper bounds with the following assumptions: the fraction of dangling ends is monotonically increasing in time and approaches $f=0.5$ for long times, the strain coupling will be isotropic in the long time limit $(\nu=0)$ and finally that 

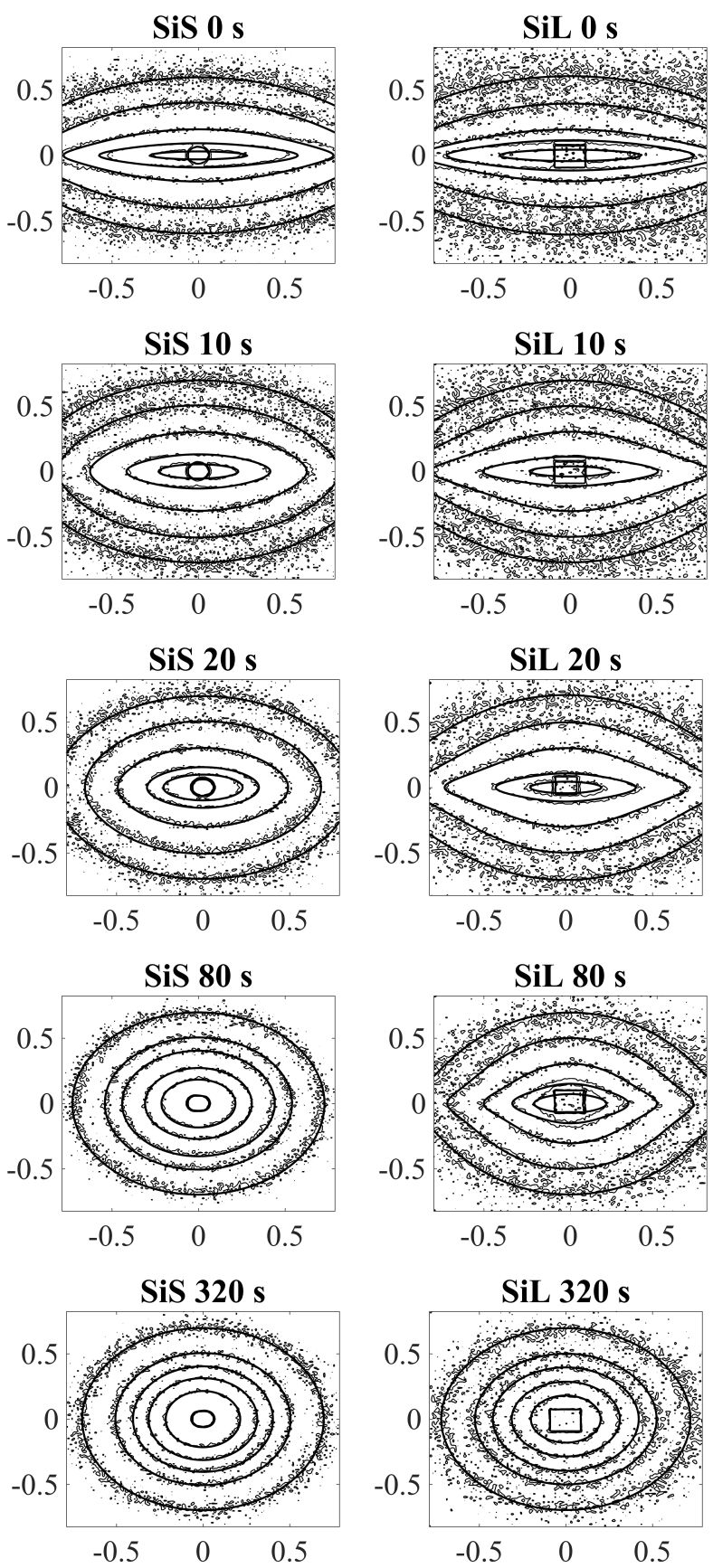

FIG. 2: Two-dimensional scattering data and accompanying fits for both sample series. We omit the totally relaxed 1260 second data. Units are in $\mathrm{nm}^{-1}$. (left) SiS sample for times 0 , 10, 20, 80 and 320 seconds. (right) Same for the SiL sample.

the effective strain felt by the short chains will be monotonically decreasing as the system relaxes. We note that we find it impossible to fit the $t=0$ data assuming affine or isotropic microscopic deformations $(\nu=1$ or $\nu=0)$ with reasonable physical parameters. In our analysis we fix $R_{g}=7.82 \mathrm{~nm}$, the value obtained from an isotropic Debye fit to the fully relaxed sample and $d_{0}=7.51 \mathrm{~nm}$, calculated from $R_{g}^{2}=d_{0}^{2} Z_{S} / 6$. The fits are done using custom-made Matlab code minimising the residuals in a least-square sense. The actual fit parameters are listed in Table II and the 2D fits are shown in Figure 2. The fitting gives the presented evolution of the main fit parameters shown in Figure 3 with suitable normalizations.

From a simple visual inspection of the 2D data in Figure 2 it is clear that the short chain relaxation is affected by the presence of the long chains immediately indicating a nematic effect of the long chains. It has previously been demonstrated with infrared dichroism and NMR in similar systems of long/short blends that the local orientational order of the two chains is identical $[24,25]$ and thus we can ascribe any difference in the principal axis parameters as originating directly from a difference of the short chain stretching. Let us first focus on the initial quench in the steady state. The effective extension ratio is $\lambda_{z \text {,eff }}=d_{z} / d_{0}=\lambda_{z}^{\nu}$ which we can compare to either the fully oriented, but unstretched chain or the fully stretched chain via the end-to-end vector. The ratios in those two cases are $Z_{S} d_{0} / \sqrt{6} R_{g} \sim 2.75$ and $N_{k} b / \sqrt{6} R_{g} \sim 10.5$ respectively [26]. The numbers for the SiS and SiL samples are $\lambda_{z \text {, eff }}^{\text {SiS }}=16.21^{0.52}=4.2$ and $\lambda_{z \text {,eff }}^{\text {SiL }}=21.72^{0.61}=6.54$. Thus, the SiS chains are stretched a factor 1.5 relative to the fully oriented chain or $40 \%$ of the fully extended chain. The SiL chains are stretched a factor 2.4 relative to the fully oriented chain or ca. $60 \%$ of the fully extended chain. So relative to the SiS sample, i.e. without the nematic field of the long chains, the short chains in the blend are stretched an extra $\sim 50 \%$. Thus, the combined effect of the flow and the nematic field of the oriented long chains not only orients and stretches the short chains but increases the experienced stretch. In fact, the SiL fit predicts a microscopic $\lambda_{z}$ value higher than the macroscopic strain ratio. These findings can only be rationalised if direct nematic interactions exist between the chains.

As the chains start to relax it becomes clear that the behaviour in the two scenarios is also quite different. If we focus on the relaxation of the SiS sample first, the fit for $t=0$ basically confirm the $\nu=0.5$ non-affine strain coupling from [21] even for the much stronger flow conditions employed here (a factor $\sim 15$ higher strain ratio). For $t>0$ the fits to the SiS data predict that at the Rouse time the system is almost relaxed and from the effective strain ratio we find that at the 320 s mark, the system has fully relaxed. For the SiL sample, the progression of events is clearly different. In the DE model, the most robust fit parameter is the dangling end fraction which effectively weighs the scattering contributions of isotropic and stretched material. In Fig. 3(a) the evolution of $f$ shows that the relaxation of the short chains are delayed in the presence of the long chains predicting that at $\tau_{R, S}$ only around $40 \%$ of the short chains have relaxed and that a population of the chains remain stretched until af- 

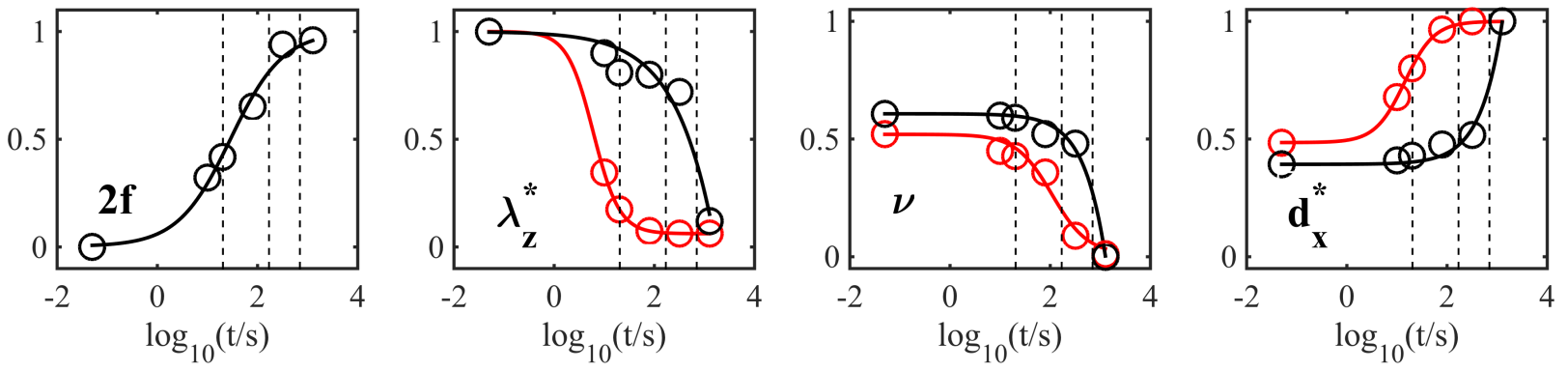

FIG. 3: Time evolution of normalized fit parameters from the DE- and WE-models: The SiL total dangling end fraction $(2 f)$, the strain ratio relative to the initial value $\left(\lambda_{z}^{*}=\lambda_{z} / \lambda_{z}(t=0)\right)$, the strain coupling parameter $(\nu)$ and the derived transverse tube diameter $\left(d_{x}^{*}=d_{x} / d_{0}=\lambda_{x}^{\nu}\right.$ where $\lambda_{x}=1 / \sqrt{\lambda_{z}}$ and $\left.d_{0}=7.51 \mathrm{~nm}\right)$. Black data is SiL, red data is SiS. Sigmoidal fits are meant as a guide for the eye. Dashed vertical lines indicate the Rouse and reptation time of the short chains (see Figure 1) and the $700 \mathrm{~s}$ found in [10] to indicate the end of a rheological transition regime $(\log (700) \sim 2.85)$.

ter $\tau_{d, S}$ and very close to the 700 s found to indicate the transition to a pure long chain relaxation in [10]. The collective output from the model fits tells the same story, namely that the effective strain is felt over a prolonged time scale in the SiL sample, but the individual fit parameters provide a much more detailed picture of how the relaxation progresses.

TABLE II: Parameters from the WE/DE-model fits.

\begin{tabular}{|c|c|c|c|c|c|}
\hline & $\mathrm{SiS}$ & & $\mathrm{SiL}$ & & \\
\hline Time & $\lambda_{z}$ & $\nu$ & $f$ & $\lambda_{z}$ & $\nu$ \\
\hline 0 & $16.21 \pm 1.8$ & $0.52 \pm 0.01$ & $0 \pm 0.01$ & $21.72 \pm 4.5$ & $0.61 \pm 0.02$ \\
\hline 10 & $5.59 \pm 0.39$ & $0.45 \pm 0.02$ & $0.16 \pm 0.02$ & $19.55 \pm 4.8$ & $0.6 \pm 0.02$ \\
\hline 20 & $2.82 \pm 0.22$ & $0.43 \pm 0.05$ & $0.21 \pm 0.02$ & $17.59 \pm 5.9$ & $0.59 \pm 0.02$ \\
\hline 80 & $1.22 \pm 0.13$ & $0.36 \pm 0.04$ & $0.33 \pm 0.04$ & $17.36 \pm 5.2$ & $0.52 \pm 0.06$ \\
\hline 320 & $1 \pm 0.1$ & $0.09 \pm 0.01$ & $0.47 \pm 0.05$ & $15.61 \pm 4.08$ & $0.48 \pm 0.05$ \\
\hline 1260 & $1 \pm 0.1$ & $0.01 \pm 0.01$ & $0.48 \pm 0.02$ & $2.59 \pm 0.88$ & $0 \pm 0.01$ \\
\hline
\end{tabular}

In the SiS sample the strain relaxation is almost done after a Rouse time, while in the SiL sample the strain persist to times again very close to the 700s mentioned above, see $\lambda_{z}^{*}$ plot in Fig. 3. Fig. 1 shows that the initial relaxation is faster in the blend than in the pure melt and in [10] it is suggested that the initial fast relaxation is from primarily stretch relaxation along the chain contour of both short and long chains. For the short chains we can now attribute this to the increased stretch which effectively will excite more higher relaxation modes. Further, in the SiL sample the derived transverse tube diameter $d_{x}$ remains at a value of roughly $d_{0} / 2$ indicating that little transverse relaxation takes place initially. Thus, the overall structural picture that emerges is that the rheological transition regime is caused by a cooperative nematic effect where the short chain stretch and orientation is maintained by the long chains and relaxes primarily along the chains, i.e. by reptation as already suggested in [10]. The long chains relax in this sea of reptating short chains until around $t=700$ s after which they relax in a solvent of re- laxed short chains but likely with a given orientation distribution which has been collectively maintained during the transition regime. As mentioned above, we ascribe the increased stretch in the blend to direct interactions between the chains. Another effect of this is manifested in the strain coupling parameter which the fits predict to initially be higher than the theoretical mean field value in the SiL sample. The value of $\nu_{\mathrm{SiL}}=0.61 \mathrm{fits}$ well with values reported for stretched long chain melts [27] analysed by Straube et al. [28]. There values of 0.63 and 0.57 are reported where the sample has tempered for $60 \mathrm{~s}$ and 600 s respectively after being stretched. There is currently no theoretical explanation for how such a scaling emerges from first principles. A final note on the appearance of lozenges which in stretched crosslinked network systems are usually observed as a precursor to the socalled 'butterfly' patterns with the general time progression: ellipses, lozenges, crosses, butterflies [24]. In our data we see a hint of something at low $q$ for the 80s SiL sample which could be the onset of a cross or butterfly pattern but we have no data from 80-320s. Comparing with similar systems investigated by Hayes et al. [24] it is reasonable for these patterns to appear at this time. In fact, their appearance validates the use of the DE-model for our non-crosslinked system as it shows that the entanglement network of the blend is sufficiently sustained over the course of the measurements.

In conclusion, we confirm the ruling out of both nondeformed and affinely deformed tubes in entangled melts akin to crosslinked networks [21]. In the pure short chain melt we confirm the non-affine mean-field result $\nu=0.5$ for the strain coupling, even subjecting the sample to a significantly increased stretch and strain rate than previously documented. We propose a non-affine strain coupling relaxation with onset around the Rouse time of the short chains. In the blend we demonstrate a nematic field effect on the short chains from the aligned and stretched long chains showing an initial increased stretch of the short chains of ca. $50 \%$ and a clear ne- 
matic effect influencing the short chain relaxation. The cooperative nematic effect explains the rheological signature from a structural perspective, particularly in the intermediate transition regime appearing after an initial fast stretch dominated relaxation. Our data also suggest an increased non-affine strain coupling in the blend which we speculate derives from direct nematic interactions not accounted for in current tube theories and as such our structural data will provide input for ongoing theoretical efforts implementing direct nematic interactions at the fundamental level.

\section{ACKNOWLEDGMENTS}

We gratefully acknowledge beamtime allocations from the Paul Scherrer Institute (Switzerland) and ANSTO (Australia).

* E-mail:jjkk@nbi.dk

[1] Bach, A., Almdal K., Rasmussen H. K. , and Hassager O., Macromolecules 36, 5174-5179 (2003).

[2] McKinley, G. H. and Sridhar T., Annu. Rev. Fluid Mech. 34, 375-415 (2002).

[3] Doi, M. and Edwards, S.F., The Theory of Polymer Dynamics, Oxford University Press, Oxford (1986).

[4] Likhtman, A.E. and McLeish, T.C.B., Macromolecules, 35(16), 6332-6343 (2002).

[5] Ianniruberto, G., Macromolecules 48, 6306-6312 (2015).

[6] Yaoita, T. Isaki, T., Masubuchi, Y., Watanabe, H., Ianniruberto, G. and Marrucci, G, Macromolecules 45, $2773 ? 2782$ (2012).

[7] Huang, Q., Alvarez, N. J., Matsumiya, Y., Rasmussen H. K. and Hassager, O., Macro Letters 2, 741-744 (2013).

[8] Wingstrand, S. L., Alvarez, N. J, Huang, Q. and Hassager, O., Physical Review Letters 115(7), 078302 (2015).

[9] Wagner, M.H., Journal of Non-Newtonian Fluid Mechanics, 222, 121-131 (2015)
[10] Hengeller, L., Huang Q., Dorokhin A., Almdal K., Alvarez N. J., and Hassager O., Rheologica Acta, 2016, 10.1007/s00397-016-0916-9

[11] Huang, Q., Mednova, O., Rasmussen H. K., Alvarez, N. J., Skov, A. L., Almdal, K. and O. Hassager, Macromolecules 46, 5026-5035 (2013).

[12] Hassager, O., Mortensen, K., Bach, A., Almdal, K., Rasmussen H.K. and Pyckhout-Hintzen, W., Rheol. Acta 51, 385-394 (2012)

[13] Warner, M and Edwards, S.F., J. Phys. A: Math. Gen.11, 8, 1649-1655 (1978).

[14] Read, D.J. and McLeish, T.C.B., Macromolecules 30, 6376-6384 (1997).

[15] Read, D.J. and McLeish, T.C.B., Phys. Rev. Lett. 79, 1, 87-90 (1997).

[16] Westermann, S., Urban, V., Pyckhout-Hintzen, W., Richter, D. and Straube, E., Phys. Rev. Lett. 80, 24, 5449 (1997).

[17] Read, D.J. and McLeish, T.C.B., Phys. Rev. Lett. 80, 24, 5450 (1997).

[18] Heinrich, G and Straube, E., Polymer Bulletin, 17, 255261, (1987)

[19] Ott, M., Prez-Aparicio, R., Schneider, H., Sotta, P. and Saalwachter, K., Macromolecules 47, 7597-7611 (2014).

[20] Straube, E., Phys.Rev. Lett., 74, 22, 4464-4467, (1995)

[21] Pyckhout-Hintzen, W., Westermann, S., Wischnewski, A, Monkenbusch, M., Richter, D., Straube, E., Farago, B. and Lindner, P., Phys. Rev. Lett., 110, 196002, (2013)

[22] Heinrich, G, Straube, E. and Helmis, G., Adv. Polym. Sci., 85, 33, (1988)

[23] Rubinstein, M. and Panyukov, S., Macromolecules, 30, 8036, (1997)

[24] Hayes, C., Bokobza, L., Boué, F., Mendes, E. and Monnerie, L., Macromolecules 29, 5036-5041 (1996).

[25] Chapellier, B., Deloche, B. and Oeser, R., J. Phys II France 3, 1619-1631 (1993).

[26] Fetters, L.J, Lohse, D.J. and Colby, R.H., Physical Properties of Polymers Handbook, 447-454 (2007)

[27] Bastide, J., Herz, J. and Boué F., J. Physique 46, 19671979 (1985).

[28] Straube, E., Urban, V., Pyckout-Hintzen, W. and Richter, D. Macromolecules, 27, 7681-7688, (1994) 